\title{
Relative Navigation in LEO by Carrier-Phase Differential GPS with Intersatellite Ranging Augmentation
}

\author{
Alfredo Renga, ${ }^{1}$ Michele Grassi, ${ }^{1}$ and Urbano Tancredi ${ }^{2}$ \\ ${ }^{1}$ Department of Aerospace Engineering, University of Naples "Federico II," Piazzale Tecchio 80, 80125 Naples, Italy \\ ${ }^{2}$ Department for Technologies, University of Naples "Parthenope," Centro Direzionale, Isola C4, 80143 Naples, Italy \\ Correspondence should be addressed to Michele Grassi; michele.grassi@unina.it
}

Received 31 August 2012; Revised 21 December 2012; Accepted 4 January 2013

Academic Editor: Paul Williams

Copyright (C) 2013 Alfredo Renga et al. This is an open access article distributed under the Creative Commons Attribution License, which permits unrestricted use, distribution, and reproduction in any medium, provided the original work is properly cited.

\begin{abstract}
Carrier-phase differential GPS (CDGPS) is a promising technology for accurate relative navigation in LEO formations of cooperating satellites, but navigation filter robustness against poor GPS geometry and noisy measurements has to be improved. This can be performed by augmenting the navigation filter with intersatellite local ranging measurements, as the ones provided by ranging transponders or GNSS-like systems. In this paper, an augmented CDGPS navigation filter is proposed for the formation of two satellites characterized by a short, varying baseline, relevant to next generation Synthetic Aperture Radar missions. Specifically, a cascade-combination of dynamic and kinematic filters which processes double-differenced code and carrier measurements on two frequencies, as well as local inter-satellite ranging measurements, is used to get centimeter-level baseline estimates. The augmented filter is validated by numerical simulations of the formation orbital path. Results demonstrate that the proposed approach is effective in preserving the centimeter-level accuracy achievable by a CDGPS-only filter also in the presence of a poor GDOP or a limited number of GPS satellites in view.
\end{abstract}

\section{Introduction}

In the recent years, the interest towards the use of satellite formations to perform many space missions, such as Earth remote sensing, observation of the universe, and Earth gravity field mapping, is increased. In these applications, the platforms can either act coordinately to synthesize a unique payload or a complement to each other so as to magnify mission scientific goals.

Formation flying offers many performance and operational advantages with respect to traditional space systems based on a single platform, but at the same time, the maintenance of a prefixed formation geometry is crucial to achieve specific mission goals. This is a challenging task, requiring the implementation of a robust communication interlink between the various platforms, as well as autonomous relative navigation and control.

Many applications require the determination of the satellite separations with centimeter-level accuracy to control the satellite relative positions, especially when they come closer as a result of the relative orbital path or at the subcentimeter level to achieve scientific goals [1]. For determining the relative position, advanced technologies based on GPS, vision, and laser systems have been proposed, and a number of missions are being performed for inflight demonstration of these technologies $[2,3]$.

With reference to LEO formations, carrier-phase differential GPS (CDGPS) is a promising technology for relative navigation. Previous studies show that processing Single difference (SD) or double difference (DD) carrier-phase measurements within dynamic filters allows determining the satellite relative positions with high accuracy. Subcentimeter-level accuracy has been demonstrated for satellite formations characterized by separations from a few kilometers to hundreds of kilometers [4-7], although only in postprocessing.

A major technical problem in implementing CDGPS is solving for the unknown integer number of cycles (integer ambiguity). Moreover, accuracy and robustness of the integer solution have to be preserved against the varying number of common-in-view satellites and ephemeris and ionospheric differential errors, especially when separations are highly 
variable, with the satellites continuously coming closer and departing from each other because of the relative orbital path. This is the case of remote sensing applications in which several formations (parallel, pendulum, and helix) are typically proposed to reflect next generation monostatic/bistatic spaceborne Synthetic Aperture Radar (SAR) mission needs in LEO $[1,8]$.

In addition, navigation filter performance and robustness can be degraded in case of poor GPS geometry, and when a reduced number of GPS measurements is available or anomalous, data are gathered. Finally, it is well known that GPS-only measurements commonly attain poor radial or vertical accuracy [9-11].

To overcome the limitations of a GPS-only navigation system, augmented CDGPS technologies can be implemented [12-15]. Specifically, local inter-satellite ranging signals can be exploited to improve accuracy and robustness of CDGPSbased relative navigation. In particular, in [13], it is demonstrated that for baselines of a few kilometers, local ranging measurements can be properly combined with GPS measurements to provide a sufficient number of measurements and adequate geometry to solve for the relative position and velocity states with high accuracy. In [14], the augmentation of CDGPS with direct ranging measurements is suggested as a way to enable more rapid and stable converge of the navigation filter solution, especially when the separation among the satellites increases as a result of the relative orbit.

The local ranging measurements can be provided by an inter-satellite Radio Frequency (RF) or optical link [12]. In particular, the RF interlink can be used also for large separations and satisfies the requirement of data distribution among the satellites of the formation. Indeed, GPS data have to be exchanged between the various satellites to form the differential GPS observables needed for CDGPS relative navigation. The RF link can be implemented by means of ranging transponders [12, 15-17] or GNSS-like signal-based systems $[12,13]$, as the one experimented in the PRISMA mission [14].

The availability of local ranging measurements not only improves the accuracy and robustness of a GPS-only relative navigation system, but also improves the accuracy in the radial component determination. Indeed, as shown in [18], an inter-satellite ranging system measuring the along-track baseline variations over the time can be, in principle, used to evaluate the differences in semimajor axis between the satellites. Any difference in semimajor axis is related to the radial separation between the satellites [19].

In previous works [20-24], the authors presented CDGPS-based filtering approaches for precise relative navigation of LEO formations characterized by a computational load compatible with real-time onboard operation requirements. These approaches rely on using an extended Kalman filter (EKF) combined in cascade with a kinematic filter. The dynamic filter outputs accurate estimates of the floating values of the DD integer ambiguities, whose integer values are extracted by the LAMBDA method [25]. They are then processed in the kinematic filter to obtain precise relative position estimates. Centimeter-to-decimeter level accuracy has been demonstrated in these works, also by application to flight data $[21,22]$, even though a degradation of the accuracy has been observed under the worst case conditions (poor GPS geometry, reduced number of satellites in common view, and large separations), especially in the radial direction.

To overcome these limitations, in the present paper, the proposed filtering approach is generalized by introducing inter-satellite link (ISL) ranging measurements in combination with the GPS ones. The benefits of fusing ISL and GPS measurements are assessed by comparing the filter performance when processing both observables to the standard GPS-only filter proposed in [20]. The filter performances are ascertained by means of numerical simulations of a formation of two satellites, a chief and a deputy, flying according to different orbital configurations characterized by varying inter-satellite distances. The worst case conditions in terms of GPS satellite observation geometry and ionosphere activity are simulated.

\section{Intersatellite Ranging Technologies}

Different technologies can be used for inter-satellite ranging. They can be classified in RF, optical or laser, and laser interferometry. In the considered scenario, however, optical or laser, metrology cannot achieve a satisfactory performance being typically characterized by limited range and/or limited field of view. RF metrology based on ISL relies on local transmission/reception of ranging signals without using the Global Navigation Satellite System (GNSS) constellations. In this context, different technologies can be potentially exploited:

(i) GNSS-like signals;

(ii) Transponders;

(iii) ultra-WideBand (UWB) signals;

(iv) wireless based.

The technology based on GNSS-like signal is undoubtedly the most mature, taking advantages of well-assessed experiences on GNSS hardware and software operational issues. These systems rely, in principle, on one-way ranging signals whose structure can be the same as conventional GPS C/A transmissions [12]. This technology is therefore influenced by classic GPS error sources and uncertainties: the ranging measurement is actually a pseudorange, including also transmitter and receiver clock biases and signal propagation delays. Carrier-phase observables can be also derived and used for ranging but only after ambiguity resolution and removal.

A ranging system, based on GNSS-like signals, was embarked, tested, and validated on PRISMA mission [13]. The system, whose operational range is a few tens of $\mathrm{km}$, is actually characterized by a dual-frequency S-band configuration, which allows performing carrier ambiguity resolution by wide-lane techniques. In addition, each transmitter is also able to receive GNSS-like signals generated by the other satellites of the formation. In this way, relative clock drifts between the platforms can be monitored. Finally, 
a multiantenna configuration allows a precise line-of-sight (LOS) determination by properly processing multiple carrierphase observables. The overall system mass is about $10 \mathrm{~kg}$ with $30 \mathrm{~W}$ power consumption. The system demonstrated $1 \mathrm{~m}$ ranging accuracy by code acquisition and $1 \mathrm{~cm}$ accuracy by processing carrier-phase observables after ambiguity resolution. Similar approaches can be found also in [26-28]. Specifically, a GNSS-like signal-based system was proposed for RF metrology in Ka band. Again, for such a system, clock biases must be calculated, and carrier-phase measurements have to be used for accurate LOS determination by multiple antenna systems.

As far as transponder-based technology is concerned, a two-satellite scenario can be taken as a reference. The intersatellite distance is determined by the two-way path delay of a signal transmitted by the first satellite, received by the second, and then transmitted back by the second satellite, usually using a different carrier, after a default, short time span. This two-way round-trip ranging technique eliminates the need for a high accurate clock synchronization. The technique, however, is still influenced by propagation delays depending on the selected carrier frequency and the considered intersatellite distance. The performance achievable by available transponders can greatly vary depending on the transponder technology and the adopted transmission protocol. An example of such a system is the Tracking and Data Relay Satellite System (TDRSS) developed by NASA and including an Sband ranging transponder [29]. In [12], the same concept is applied to LEO formations, and a system design is presented able to guarantee $30 \mathrm{~cm}$ ranging accuracy up to $1000 \mathrm{~km}$ baselines with reduced mass $(4 \mathrm{~kg})$ and power consumption (about $10 \mathrm{~W}$ ). If an orbital propagator is used, also lineof-sight (LOS) estimations can be performed. A different approach was developed in [15] where a transponder is presented based on long code sequences (P-code like) modulated on $\mathrm{Ku}$-band signals able to achieve $1 \mathrm{~cm}$ ranging accuracy, thus avoiding ambiguity resolution issues. The operational range of such a system is limited to a few kms. Likewise, a transponder-based system for ranging measurement proposed in [30] is able to guarantee $5 \mathrm{~cm}$ ranging accuracy by code only measurements. Specifically, short code sequences are used for signal tracking and locking, whereas long-code sequences guarantee high accuracy ranging measurements. For completeness, it is important to point out that even though range measurements derived by transponders are not affected by clock biases, the synchronization of those data to a common mission time reference (e.g., GPS time) could also involve managing clock biases.

UWB ranging techniques involve the transmission of short electromagnetic pulses via a current radiating antenna. These short pulse width signals (less than $1 \mathrm{~ns}$ pulse width) have very large signal bandwidths $(1-2 \mathrm{GHz})$. In contrast to most continuous wave ranging techniques, ultra-wideband signals have no carrier and are inherently spread spectrum, making them more immune to multipath errors due to interference from a reflected carrier wave signal. In [31], a UWB ranging system is developed and tested in an anechoic chamber. The experimentation showed the possibility to guarantee a sub-centimeter ranging accuracy also eliminating the measurement bias ambiguity problem. However, important limitations still remain which prevent the implementation of such a technology in satellite formation flying applications, including the design of UWB antennas and spectrum allocation and pollution.

Among wireless protocols, the most promising is the 802.16 e, able to guarantee long-range communications (up to $10 \mathrm{~km}$ ) [12]. However, this standard is not space qualified yet.

From the reported analysis, some considerations can be drawn. To satisfy the requirement of autonomous relative navigation and control of LEO formations, the ISL system has to be used for augmenting the CDGPS in real-time implementation. Therefore, the selected ISL system must be simple, sufficiently accurate, and robust. This means that only range measurements are considered for CDGPS augmentation. This actually excludes carrier-based techniques which are very accurate but are not simple to implement and in general not robust due to the ambiguity resolution issues. In this context, both one-way (GNSS-like) and twoway (transponders) code-based techniques represent the best choice to provide the navigation algorithm with robust decimeter accuracy ranging measurements in a simple way. Different technologies, such as those using UWB signals or wireless-based signals, are very promising but, at the present time, neither mature nor robust enough to be considered for the relative navigation in satellite formation flying missions.

\section{Augmented Filter Model}

The filter model processing both CDGPS and ISL measurements represents an extension of the filter presented in [20], based on the results reported in $[4,7]$, developed for a formation of two satellites, named chief and deputy, in the Earth-Centered-Earth-Fixed (ECEF), and using only GPS carrier-phase and pseudorange observables.

As well known, the relative navigation problem is well described by the following nonlinear discrete stochastic model:

$$
\begin{gathered}
x_{n+1}=g_{n}\left(x_{n}\right)+v_{n}, \\
y_{n}=h_{n}\left(x_{n}\right)+w_{n},
\end{gathered}
$$

where $x$ is the system state vector, $y$ is the measurement vector, $g$ is the non-linear state propagation function, $h$ is the nonlinear observation function, $v$ is the process noise vector, $w$ is the measurement noise vector, and the subscript $n$ is used to denote the variable value at the time $t_{n}$. Both noises are assumed to be additive, white, Gaussian with zero mean, uncorrelated in time, mutually uncorrelated, and uncorrelated with the state vector at the same time sample. The following covariance matrixes for the variables defined in (1) are introduced: $P_{x x}$ for $x, P_{y y}$ for $y, Q$ for $v$, and $R$ for $w$. With respect to the implementation presented in [20], the measurement model now includes the range measurement $D$ in addition to the dual-frequency, DD pseudorange, and 
carrier-phase observables. The state and the measurement vectors are thus given by

$$
\begin{aligned}
& x=\left(\begin{array}{llllll}
\underline{B} & \underline{B} & \mathrm{VTEC}_{1} & \mathrm{VTEC}_{2} & L_{1}^{\mathrm{AM}} & L_{2}^{\mathrm{AM}}
\end{array}\right)^{T}, \\
& y=\left(\begin{array}{lllll}
L_{1}^{\mathrm{PR}} & L_{2}^{\mathrm{PR}} & L_{1}^{\mathrm{CP}} & L_{2}^{\mathrm{CP}} & D
\end{array}\right)^{T},
\end{aligned}
$$

where

$$
\begin{aligned}
& x \in \mathbb{R}^{\left[8+2\left(N_{\mathrm{sat}}-1\right)\right] \times 1}, \\
& y \in \mathbb{R}^{\left[4\left(N_{\mathrm{sat}}-1\right)+1\right] \times 1},
\end{aligned}
$$

and $N_{\text {sat }}$ is the number of commonly viewed GPS satellites, $\underline{B}$ is the baseline vector, $\underline{B}$ is the relative velocity, $\mathrm{VTEC}_{1}$ and $\mathrm{VTEC}_{2}$ are the vertical total electron content from, respectively, the chief and deputy receivers to the upper bound of the ionosphere, $L_{1}^{\mathrm{AM}}$ and $L_{2}^{\mathrm{AM}}$ are the DD integer ambiguity vectors on the $L_{1}$ and $L_{2}$ carriers, respectively, $L_{1}^{\mathrm{PR}}$ and $L_{2}^{\mathrm{PR}}$ are the DD pseudorange measurements, and $L_{1}^{\mathrm{CP}}$ and $L_{2}^{\mathrm{CP}}$ are the DD carrier-phase measurements. It is worth noting that a double difference can be formed by subtracting two single difference equations of the same type and frequency, taken by the same two GPS receivers at the same time from two different GPS satellites, one of which, named pivot, is taken as a reference. The pivot satellite is selected as the one with the highest elevation with respect to the chief.

Non-linear Keplerian equations have been selected for modeling the satellites' relative motion in ECEF [20, 32]. The selected model has to be interpreted as a trade-off between using a nonlinear model to improve accuracy and having a computational load adequate to real-time implementation. In this way, it is possible to get good performance by modeling the small differential perturbations (e.g., differential J2 and drag) as process noise while keeping low the computational effort [32]. Indeed, formation flying satellites usually lie on orbits whose parameters slightly differ and have similar ballistic coefficients so as to minimize control efforts to maintain the formation. VTEC terms and carrier-phase ambiguities are treated as random walk plus random constant processes $[4,7$, $20,32]$. It is worth noting that, once fixed, the carrier-phase ambiguities do not change with time, in the absence of cycle slips. Thus, the random constant model should be considered as exact for these bias terms. However, introducing a small amount of process noise on carrier-phase ambiguities allows keeping the filter receptive to new measurements, making it able to update these bias terms over the time, even if the convergence of filter covariance matrix has been already achieved [5].

3.1. Local Ranging Measurement Model. For the sake of deriving the non-linear observation function of the augmented filter and the relevant Jacobian matrix, an observation model for the ISL ranging measurement must be introduced. In general, for both transponders and GNSS-like systems operating in LEO orbits, the ranging measurements can be affected by time-of-flight (TOF) errors and ionospheric delays. The time of flight correction accounts for the motion of the platforms during signal travel time. Actually, for any given instant of reception of the ranging signal, the measured range does not represent the true baseline at that time instant but the distance between the transmitting platform at the instant of transmission and the receiving platform at the instant of reception. The difference between the desired baseline and the measured distance depends on the signal time of flight and on platforms velocity. To a first order, it can be estimated as the ratio between the baseline and platform velocity; this means that if only short separations are considered $(1-2 \mathrm{~km}$ at most), the expected TOF error is on sub-centimeter scale. The ionospheric delay, IO, depends on the electron density $\rho_{\mathrm{e}}$ at the considered LEO altitude [33]. Consider

$$
I O=\frac{40.3}{f^{2}} \int_{0}^{B} \rho_{\mathrm{e}} d B,
$$

where $f$ is the carrier frequency in $\mathrm{Hz}$, and lengths must be expressed in meters. A typical electron density value for $450 \mathrm{~km}$ altitude is $0.5 \cdot 10^{11}$ particle $/ \mathrm{m}^{3}$ [33], thus leading to a ionospheric delay of less than $0.1 \mathrm{~cm}$ for S-band operation at $2 \mathrm{~km}$. Lower electron densities are experienced at higher altitudes.

According to those considerations, for the selected orbital scenario (see Section 4) with limited inter-satellite separations, the following observation model can be assumed for the ISL ranging measurements:

$$
D=\|\underline{B}\|+\sigma_{D},
$$

where $\sigma_{D}$ represents the measurement noise. Therefore the Jacobian matrix of the observation model, $H,[4,7,20]$ must be modified by the addition of the relevant line

$$
\left(\begin{array}{lllll}
\frac{B_{X}}{\|\underline{B}\|} & \frac{B_{Y}}{\|\underline{B}\|} \quad \frac{B_{Z}}{\|\underline{B}\|} & 0_{1 \times 5} & 0_{1 \times 2\left(N_{\text {sat }}-1\right)}
\end{array}\right) .
$$

In addition, a purely diagonal term accounting for the variance of the ranging measurement must be added to the standard DD measurement noise matrix $R[4,7,20]$. Finally, it is essential to point out that the additional ranging measurement is used both in the EKF and in the kinematic filter processing only carrier-phase measurements corrected for integer ambiguities.

3.2. Navigation Filter Implementation Logic. Figure 1 shows a conceptual representation of the filtering scheme. At the generic time step $n$, the filter receives in input the estimates of the state vector and covariance matrix, $P_{x x}$, at the previous step, along with the GPS observables from chief and deputy satellites and the local ranging measurements. A coarse evaluation of the chief satellite ECEF position and velocity has to be provided as well. Measurements from commonly available GPS satellites are extracted in order to calculate DD observables. Since both the number of commonly viewed GPS satellites and the pivot satellites (i.e., the reference satellite to calculate DD observables) can change between subsequent observations, a rearranging/reinitialization step is needed to correctly relate the integer ambiguities that are part of the state vector to the current measurements [7]. 


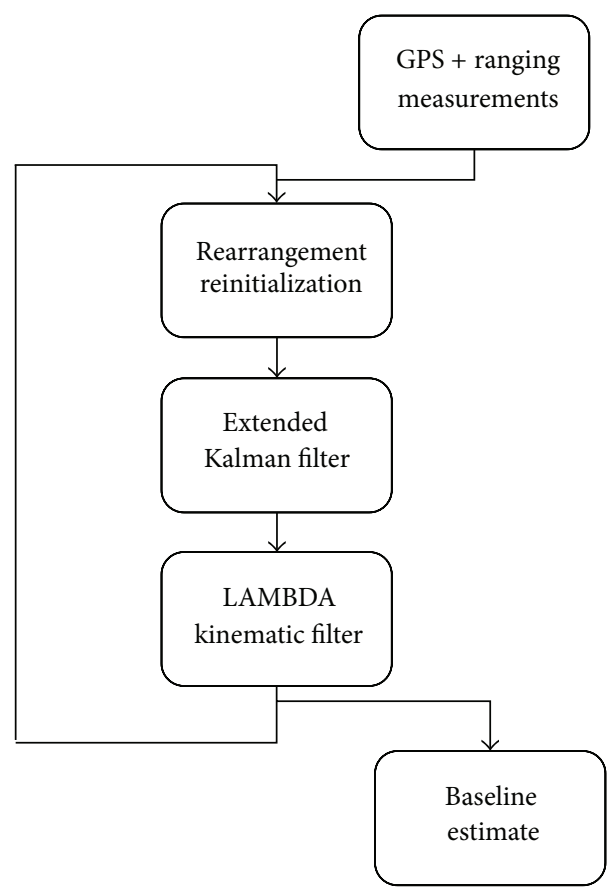

FIGURE 1: Block diagram of the CDGPS-only filtering scheme $[4,7$, 20].

The rearranged state vector and covariance matrix are used as inputs to an extended Kalman filter (EKF). The purpose of using the EKF is to perform a robust floating estimate of the DD ambiguities, whose integer values are then extracted by the LAMBDA method $[7,20]$. This allows correcting the individual DD carrier phase observations, which can be then used within a kinematic approach based on a Weighted Least Square (WLSQ) algorithm to produce a highly accurate baseline estimation $[4,7,20]$. Residual errors in the carrier phase observables depend mainly on DD ionosphere path delays estimation errors. A common procedure to eliminate this effect is evaluating ionosphere free combinations of corrected carrier phase observables [10]. It is worth noting that the cascading of an EKF with a kinematic solver can represent a potential source of instability, because the measurements used by the kinematic solver are already filtered by the EKF [10]. However, this is not considered harmful in the present case, because once DD integer ambiguities are correctly resolved (by LAMBDA), they become known deterministic parameters when used to un-bias DD carrier phase measurements. As a consequence, they do not have time correlation, and they are uncorrelated with one another. These considerations relax notably any potential risk of instability.

Finally, some remarks are needed about clock biases and time referencing. The considered GPS-based filtering scheme does not need to account for clock biases as they are removed when DD combinations are formed. Anyway, clock biases are still important to synchronize GPS measurements and to align ranging measurements with GPS ones. In the present case, the strategy reported in [34] is assumed. Actually, accurate estimations of clock biases are
TABLE 1: Parameters of the selected helix formation.

\begin{tabular}{lc}
\hline Parameter & Value \\
\hline Semimajor axis $(\mathrm{km})$ & 6997.94 \\
Eccentricity & 0.00118 (chief) -0.001308 (deputy) \\
Inclination (deg) & 97.87 \\
Anomaly of perigee (deg) & 90 \\
Right ascension of ascending & 0 (chief) -0.0049 (deputy) \\
node (deg) & 0 \\
Initial mean anomaly (deg) & \\
\hline
\end{tabular}

a standard result produced by GPS receivers. Concerning this, it is not necessary to generate a new estimate of the clock biases because it is possible to exploit the estimates that are automatically computed by the receivers. These solutions represent the starting point to establish a common time frame between the satellites and the relevant measurements too. Actually, as shown in [34], raw GPS measurements can be transformed to integer seconds of GPS time resulting in synchronized undifferenced measurements prior to using them in the relative navigation filter. The same approach holds also for the ranging measurements.

\section{Numerical Results}

Filter performance has been numerically evaluated by using the Satellite Navigation Toolbox 3.0 for Matlab developed by GPSoft, Inc. [35]. The toolbox is capable of simulating the GPS satellite constellation, the propagation environment, and the receiver measurements, where the nominal GPS satellite constellation [35], based on 24 satellites distributed on 6 orbital planes, has been assumed.

Since the Satellite Navigation Toolbox was developed mainly for Earth-based GPS receivers, a major modification to the ionosphere delay model has been introduced to adequately model first- and second-order ionosphere delay terms for LEO satellites [36]. In addition, the toolbox implicitly assumes a constant value for the VTEC. In order to overcome this limitation, the toolbox has been complemented with IONosphere map Exchange file format (IONEX) reader. Indeed, VTEC maps are provided by the international GPS service as IONEX files [37]. Unfortunately, these maps are relevant only to ground-based users. Nevertheless, for the sake of using IONEX products to estimate the VTEC behaviour along the orbit, a coefficient can be defined to scale the IONEX VTEC values to an LEO receiver as in [38].

Filter performance is evaluated by comparison with a simulated truth obtained by running an orbit propagator including a seven-order model of the gravity field and the aerodynamic drag and with reference to orbit scenarios relevant to Earth remote sensing applications. Specifically, a helix-like [36] configuration is considered relevant to interferometric and bistatic SAR applications, such as the generation of digital elevation model (DEM) by SAR interferometry.

As shown in Table 1, the considered orbits share inclination, semi-major axis, and anomaly of perigee. Figure 2 


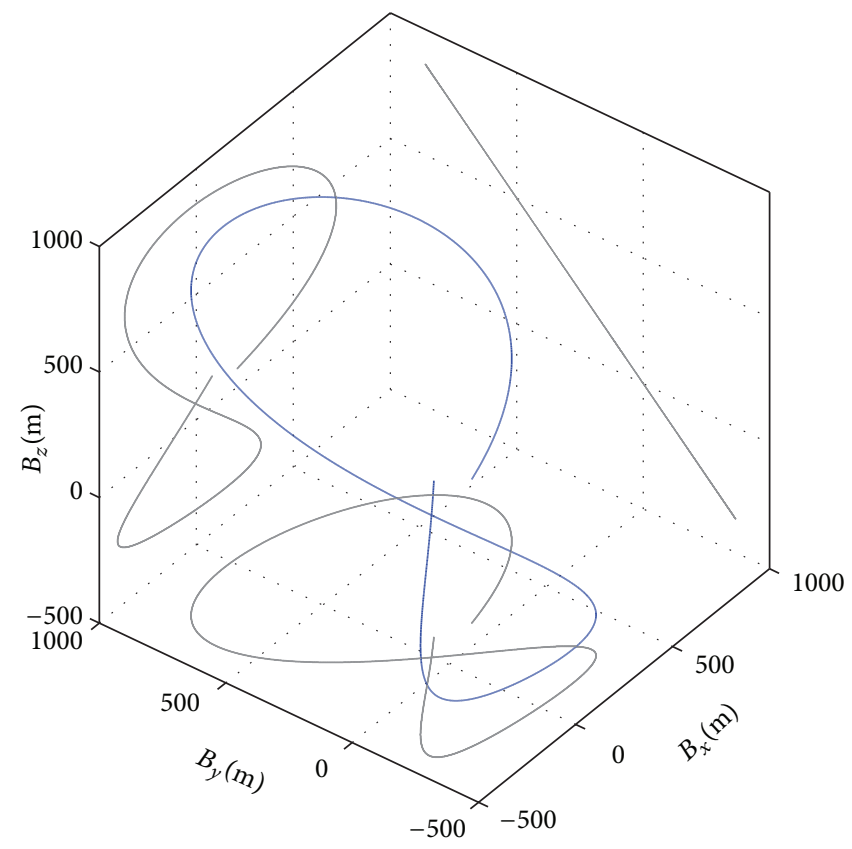

FIgURE 2: Variation of the baseline in ORF during a single orbit for the selected helix formation.

reports the variation of the baseline along one orbit for the considered helix formation with respect to the chief satellite Orbiting Reference Frame (ORF), whose origin is in the chief satellite center of mass; the $x$-axis is directed along chief velocity, and the $z$-axis is directed towards the Earth's center. It is worth noting that the axes of the ORF are computed considering the velocity of the chief satellite in ECEF; for this reason, the familiar helix shape cannot be recognized from Figure 2.

In all the simulation, the Italian COSMO-SkyMed mission is taken as a reference for the master satellite, and present generation GPS receiver nominal performance is considered, specifically $0.2 \mathrm{~m}$ and $0.25 \mathrm{~m}$ pseudorange measurement noise on $L_{1}$ and $L_{2}$ channels, respectively, and $0.5 \mathrm{~cm}$ carrier phase noise both on $L_{1}$ and $L_{2}$. In addition, $1 \mathrm{~Hz}$ GPS data frequency update and $10^{\circ}$ mask angle are considered. Finally, ISL ranging measurements are simulated considering $10 \mathrm{~cm}$ measurement noise. No meaningful propagation effects are expected for the ISL system due to the selected helix configuration characterized by limited inter-satellite separations. The chief satellite is supposed to receive deputy GPS observables by means of the ISL. The filter is supposed to run on the processing unit of the chief satellite.

All the results are derived with reference to a single orbit scenario. The considered GPS satellite geometry and ionosphere status are the same selected in [20], representing an example of a worst case condition characterized by very unfavorable GPS geometries, with geometric dilution of precision (GDOP) up to 18 , and anomalous ionosphere conditions, with VTEC up to 12 TECU (Total Electron Content Unit, corresponding to $10^{16}$ electrons per square meter) as reported in Figures 3 and 4. It is important to point out that due to the short inter-satellite separation (less than $1.5 \mathrm{~km}$ at most), VTEC values for master and slave satellites are very close, With the maximum difference being less than 0.1 TECU.

The performance of the CDGPS augmented filter presented in this paper is compared to that obtained by the GPSonly filter in [20]. The two filters share the same structure, but the latter does not use ISL ranging measurements. Before presenting the results, it is essential to point out that when GPS satellite geometry is good in terms of both GDOP and a number of common satellites and when the filters are able to correctly estimate integer ambiguities, the results must be necessarily very similar, since the accuracy of the baseline calculated by the kinematic algorithm is essentially determined by carrier-phase measurements, that are one order of magnitude more accurate than ISL ones.

Two cases have been then selected as representative of the improvement achievable by CDGPS augmentation. The same nominal parameters are simulated in the two cases, but different realizations of the measurement noises are generated. In the first case, both filters are able to correctly fix integer ambiguities; however, due to the fact that there are only 4 common GPS satellites (see Figure 3), the CDGPS-only filter cannot fully exploit the kinematic algorithm, with an evident growth of the baseline error. Specifically, the baseline norm exhibits a maximum error larger than $0.85 \mathrm{~m}$ (Figure 5(a)). This error is determined by the errors in the radial and along-track components of the baseline, with the radial error contributing for the most part as shown in Figure 6. As expected, the possibility of using an additional ranging 


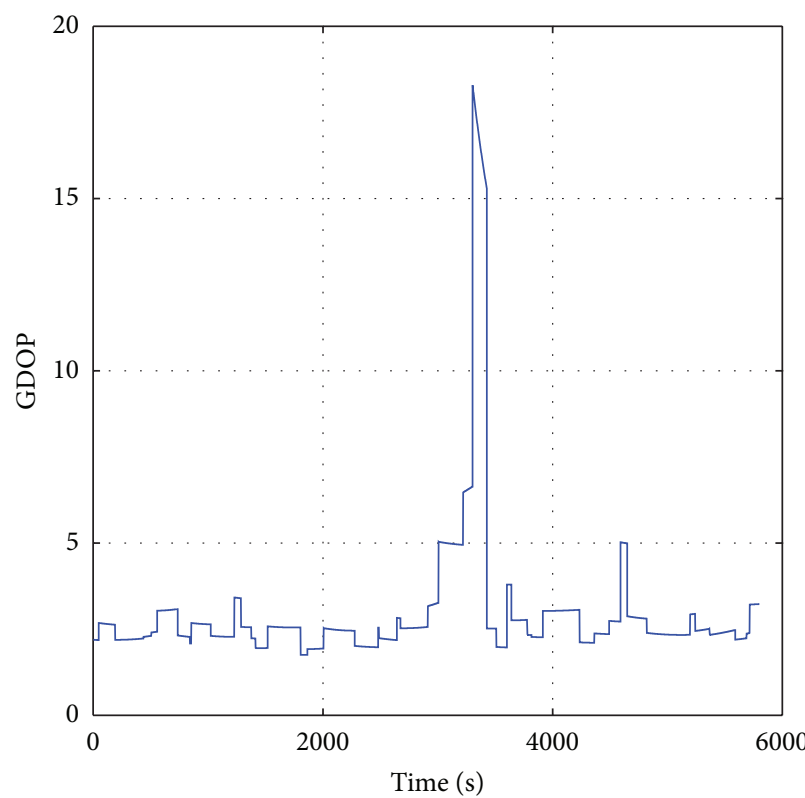

(a)

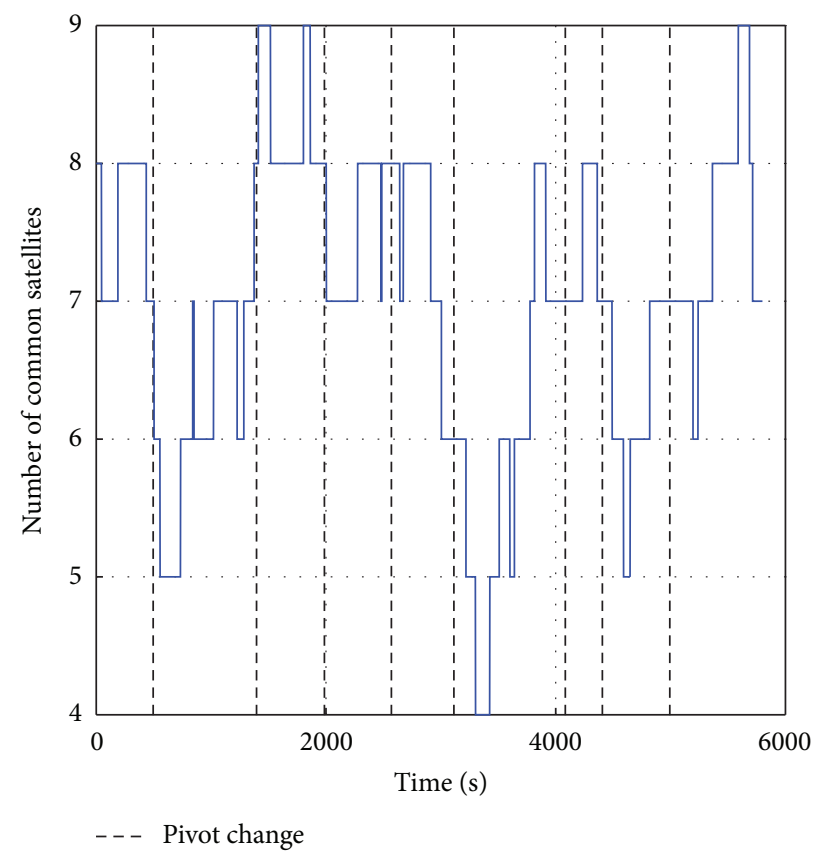

(b)

FIGURE 3: GDOP (a) and a number of common GPS satellites (b) for the selected single orbit scenario.

TABle 2: Performance statistics (the first case).

\begin{tabular}{lcccc}
\hline & \multicolumn{2}{c}{ CDGS only } & \multicolumn{2}{c}{ CDGDS + ILS } \\
& RMS error, cm & Max. error, cm & RMS error, cm & 3 \\
Max. error, cm \\
\hline Baseline norm & 5 & 36 & 2 & 17 \\
Along-track component $\left(B_{x}\right)$ & 2 & 30 & 1 & 8 \\
Cross-track component $\left(B_{y}\right)$ & 2 & -7 & 4 & 7 \\
Radial component $\left(B_{z}\right)$ & 6 & -97 & 4 & 17 \\
\hline
\end{tabular}

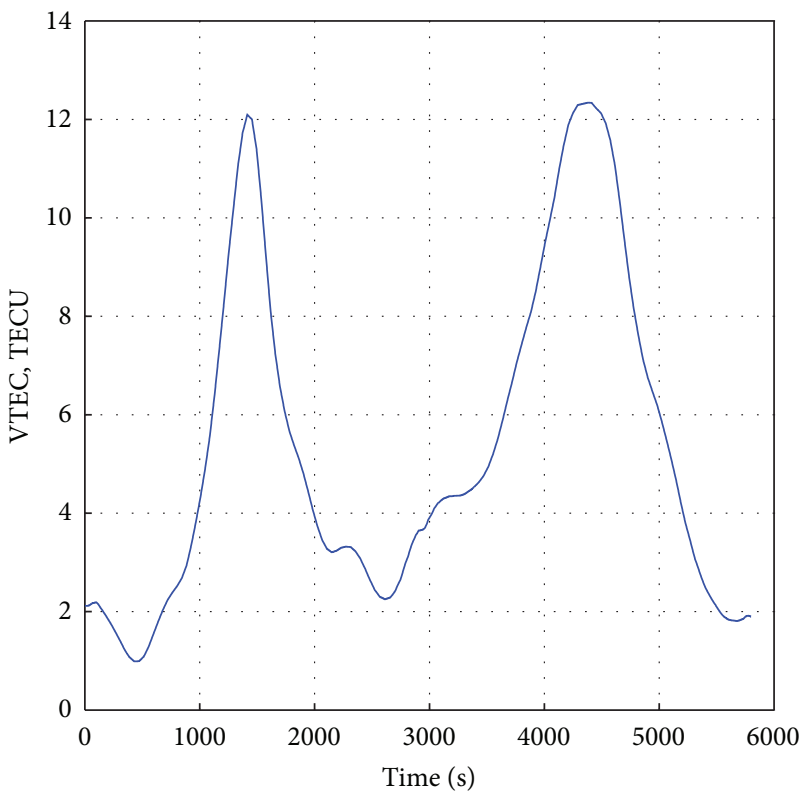

Figure 4: Simulated VTEC time profiles for the chief satellite. measurement can be properly exploited by the present filter structure, thus reducing notably both the vertical and alongtrack estimation errors and allowing the filter solution to be less sensitive to GPS satellite geometry. Figure 6 also reports the formal errors, $\pm 3 \sigma$ bounds, estimated from the covariance of the kinematic filter. The strong agreement between the formal error and the achieved one is manifest.

Table 2 summarizes filters' performance. The utilization of ranging measurements reduces both the maximum baseline norm error and the maximum radial component error by a factor of five. In addition, the maximum along-track error is reduced by a factor close to four, and also all the rms errors improve. Finally, it is worth noting that when only 4 common GPS satellites are available, the CDGPS-only filter cannot derive an estimate of the filter error covariance by the kinematic algorithm, whereas the additional ranging measurement (Figure 6) guarantees proper covariance estimations.

In the second case, characterized by worse measurement noise conditions, the filter with no range measurements is not able to correctly fix integer ambiguities in the presence of a poor GPS satellite geometry. Specifically, a wrong integer 


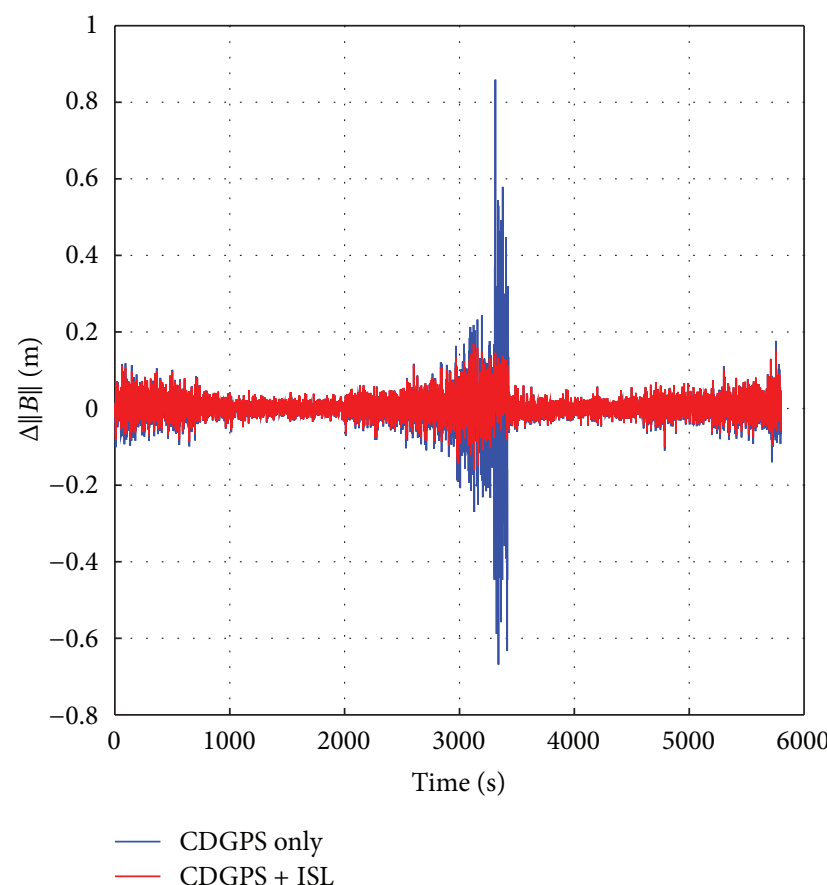

(a)

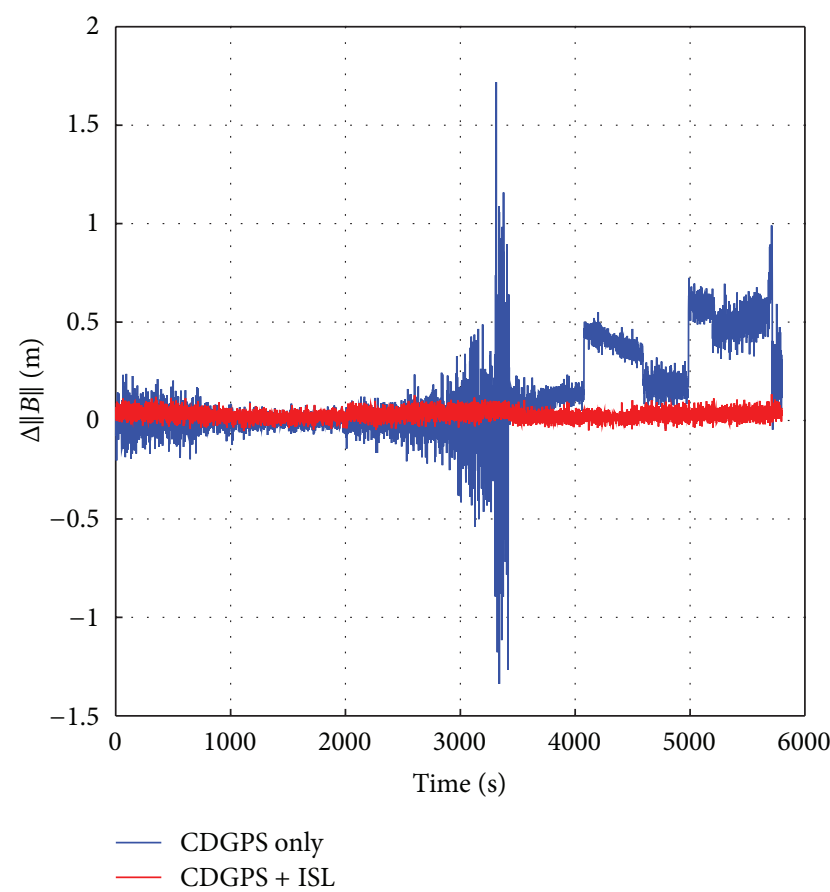

(b)

FIgURE 5: (a) Baseline norm error (the first case). (b) Baseline norm error (the second case).

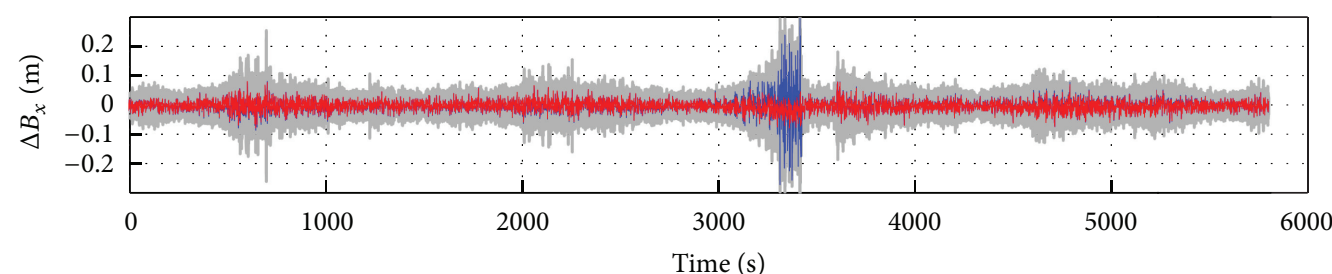

(a)

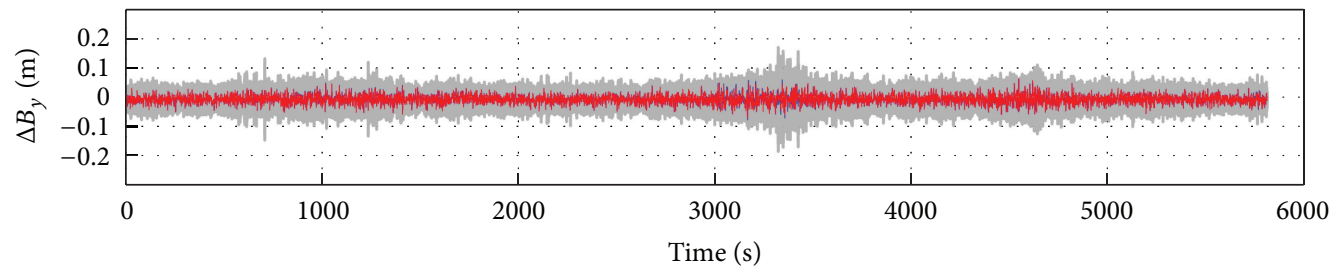

(b)

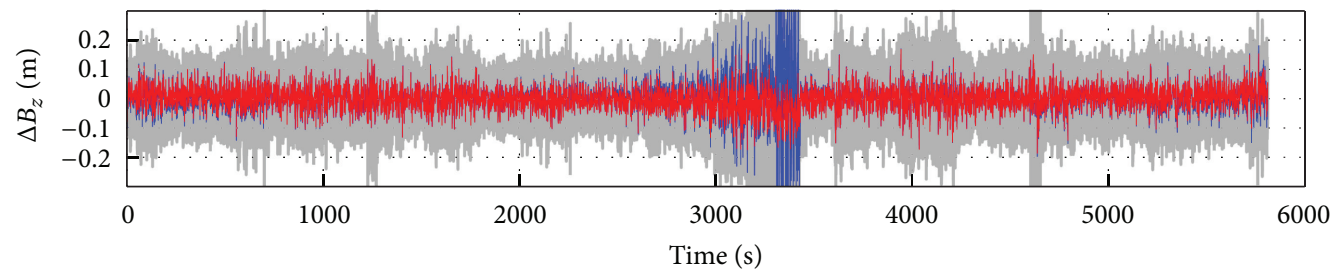

$3 \sigma$ bounds

— CDGPS only

— CDGPS + ISL

(c)

FIGURE 6: ORF baseline components error (the first case); $\pm 3 \sigma$ bounds are relevant to CDGPS + ISL. 


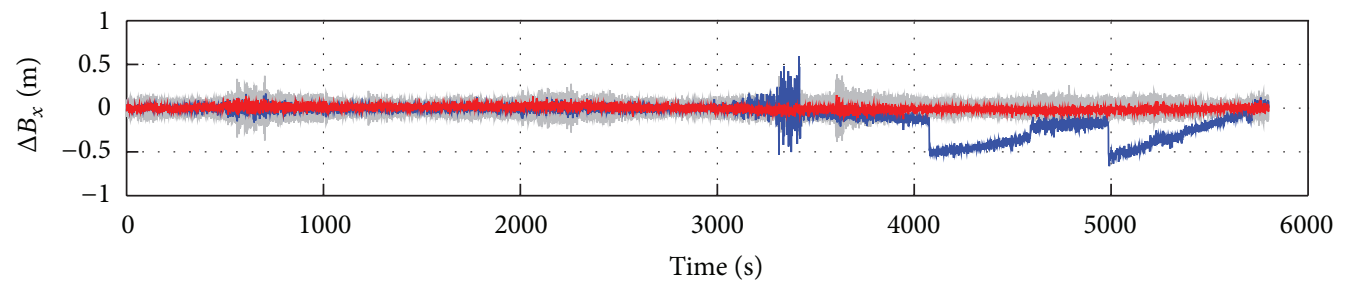

(a)

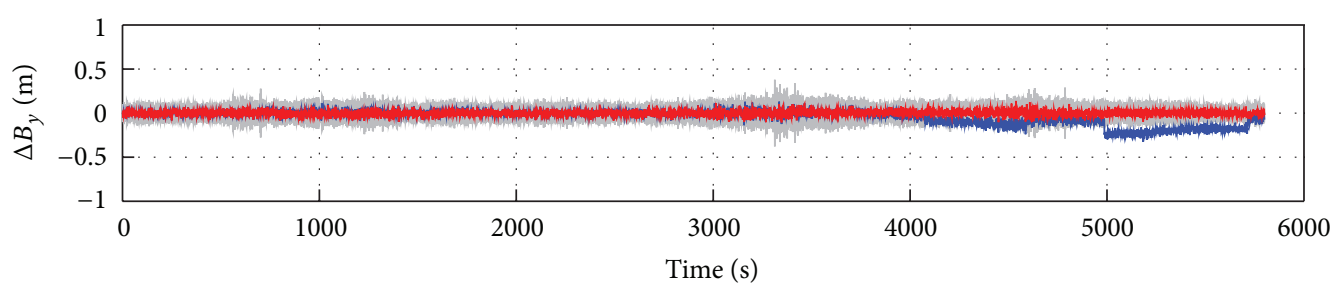

(b)

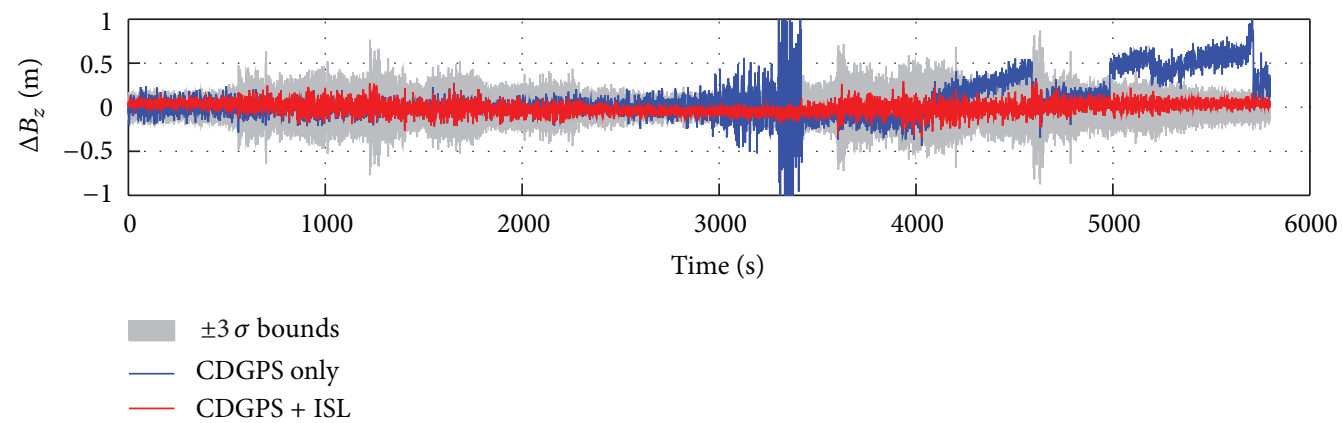

(c)

FIGURE 7: ORF baseline component errors (the second case); $\pm 3 \sigma$ bounds are relevant to CDGPS + ISL.

ambiguity value is derived around $t=3300 \mathrm{~s}$. In the following time instants, even if the GDOP improves, the filter is not able to recover good ambiguity estimates, and a diverging behavior can be thus observed in Figures 5(b) and 7. In the same condition, in the CDGPS-augmented filter, the ranging measurement effectively aids the integer ambiguity estimation process, thus preventing the diverging behavior observed in the CDGPS-only filter. Performance statistics for this second case are not reported since those of the CDGPS-augmented filter are very similar to the previous case, while the performance statistics of CDGPS-only filter are strongly influenced by wrong integer ambiguities and filter divergence. Concerning this, it is important to remark that when ambiguities are not fixed correctly, the formal error of the kinematic filter is not able to represent actual error (Figure 7).

Finally, Figure 8 shows the DD ionospheric delays as estimated by the considered filters. When wrong ambiguities are computed, the CDGPS-only filter overestimates the ionospheric delays, thus preventing the filter to correctly estimate the following ambiguities. The availability of ranging measurements, instead, allows the filter to adequately compute DD ionospheric delays, even though their order of magnitude is close to the carrier-phase noise.

\section{Conclusion}

In this paper, an approach to improve accuracy and robustness of CDGPS-based relative navigation of LEO satellite formations has been proposed. The approach relies on the augmentation of CDGPS with local intersatellite ranging measurements to overcome the limitations of CDGPS-only filters, whose performance is degraded by poor GPS geometries and GPS satellite visibility, as well as by noisy measurements. The proposed filtering scheme processes doubledifference code and carrier-phase GPS measurements on two frequencies as well as local ranging measurements provided by an inter-satellite radio link with decimeter-level accuracy. Effectiveness and performance of the proposed augmented filter have been verified by numerical simulations of LEO satellite formations relevant to next generation SAR missions characterized by short (a few kilometers), variable satellite separations. Results demonstrate that the proposed approach allows preserving the CDGPS-only filter capability of correctly computing the double-difference integer ambiguities 


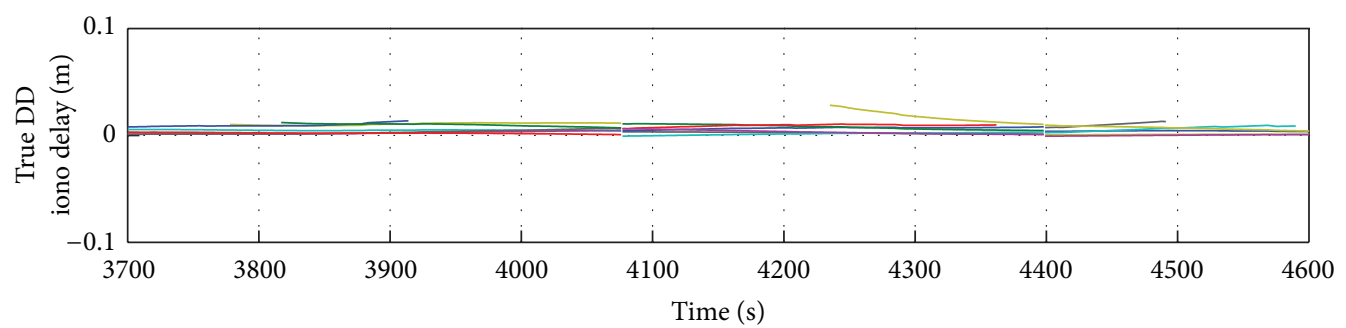

(a)

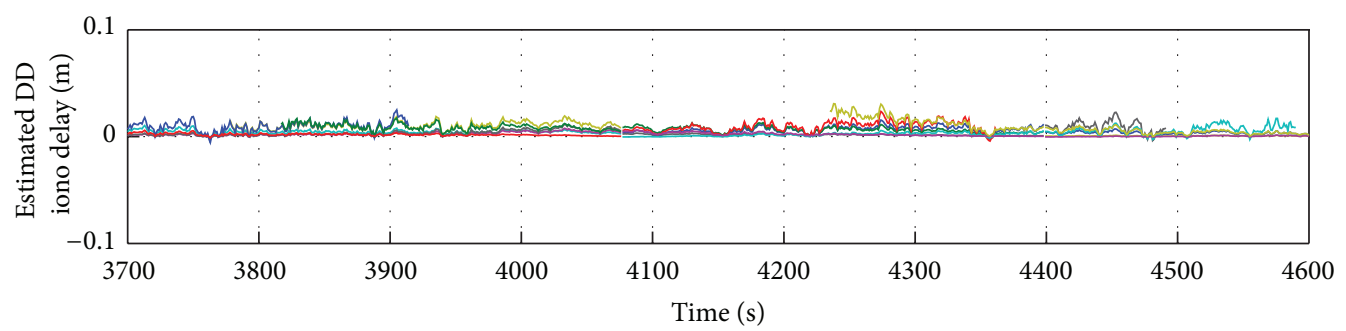

(b)

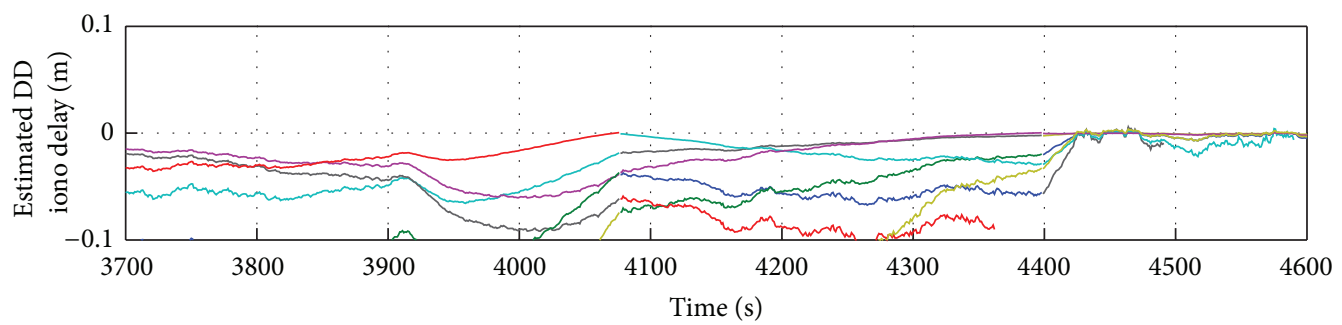

(c)

FIGURE 8: True DD ionospheric delays (a) versus estimated DD ionospheric delay by CDGPS + ISL (b) and CDGPS only (c).

also in the presence of poor GDOP. In addition, also when ambiguities are correctly computed, the availability of ranging measurements improves the accuracy in the estimate of the baseline radial component.

\section{Conflict of Interests}

The authors declare that they have no direct financial relation with the commercial identity mentioned in the paper that might lead to a conflict of interests for any of them.

\section{Acknowledgment}

A. Renga's work in this activity has been carried out with the financial contribution of Regione Campania in the framework of the Project for Technology Innovation of Transport Systems (INSIST).

\section{References}

[1] G. Krieger and A. Moreira, "Spaceborne bi- and multistatic SAR: potential and challenges," IEE Proceedings: Radar, Sonar and Navigation, vol. 153, no. 3, pp. 184-186, 2006.
[2] S. Persson, P. Bodin, E. Gill, J. Harr, and J. Jorgensen, "Prisma an autonomous formation flying mission," in Proceedings of the 4S Symposium: Small Satellite Systems and Services, pp. 25-29, Sardinia, Italy, September 2006.

[3] G. Fasano, M. Grassi, and D. Accardo, "A stereo-vision based system for autonomous navigation of an in-orbit servicing platform," in Proceedings of the AIAA Infotech at Aerospace Conference and Exhibit and AIAA Unmanned Unlimited Conference, Seattle, Wash, USA, April 2009, art. number 2009-1934.

[4] S. Leung and O. Montenbruck, "Real-time navigation of formation-flying spacecraft using global-positioning-system measurements," Journal of Guidance, Control, and Dynamics, vol. 28, no. 2, pp. 226-235, 2005.

[5] T. Ebinuma, R. H. Bishop, and E. G. Lightsey, "Integrated hardware investigations of precision spacecraft rendezvous using the global positioning system," Journal of Guidance, Control, and Dynamics, vol. 26, no. 3, pp. 425-433, 2003.

[6] E. Gill and H. Runge, "Tight formation flying for an alongtrack SAR interferometer," Acta Astronautica, vol. 55, no. 3-9, pp. 473-485, 2004.

[7] R. Kroes, O. Montenbruck, W. Bertiger, and P. Visser, "Precise GRACE baseline determination using GPS," GPS Solutions, vol. 9, no. 1, pp. 21-31, 2005.

[8] G. Krieger, A. Moreira, H. Fiedler et al., "TanDEM-X: a satellite formation for high-resolution SAR interferometry," 
IEEE Transactions on Geoscience and Remote Sensing, vol. 45, no. 11, pp. 3317-3341, 2007.

[9] G. T. French, UnderstAnding the GPS: An Introduction to the Global Positioning System, GeoResearch, Bethesda, Md, USA, 1996.

[10] J. Farrel and M. Barth, The Global Positioning System and Inertial Navigation, McGraw-Hill, New York, NY, USA, 1999.

[11] J. J. Spilker, "Fundamentals of signal tracking theory," in Global Positioning System: Theory and Applications, B. W. Parkinson and J. J. Spilker, Eds., vol. 1, pp. 245-327, American Institute of Aeronautics and Astronautics Inc, Washington, DC, USA, 1996.

[12] A. Garcì-Rodriguez, "Formation flight radio-frequency metrology," ESA Technical Note TEC-ETN/20007.04, Issue 1, 2007.

[13] C.-W. Park, Precise relative navigation using augmented CDGPS [Ph.D. thesis], Stanford University, Department of Mechanical Engineering, Cambridge, Mass, USA, 2001.

[14] S. Persson, S. Veldman, and P. Bodin, "PRISMA-A formation flying project in implementation phase," Acta Astronautica, vol. 65, pp. 1360-1374, 2009.

[15] R. G. Zenick and K. Kohlhepp, "GPS micro navigation and communication system for clusters of micro and nanosatellites," in Proceedings of the IEEE Aerospace Conference, vol. 5, pp. 25152522, Big Sky, Mont, USA, March 2001.

[16] P. A. Stadter, A. A. Chacos, R. J. Heins, G. T. Moore, E. A. Olsen, and M. S. Asher, "Confluence of navigation, communication, and control in distributed spacecraft systems," in Proceedings of the IEEE Aerospace Conference, vol. 2, pp. 563-578, Big Sky, Mont, USA, March 2001.

[17] J. C. Adams, W. Gregorwich, L. Capots, and D. Liccardo, "Ultra-wideband for navigation and communications," in IEEE Aerospace Conference, vol. 2, pp. 785-792, Big Sky, Mont, USA, March 2001.

[18] D. Maessen and E. Gill, "Relative orbital element estimation and observability analysis for formation flying satellites using inter-satellite range measurements only," in Proceedings of the AIAA Guidance, Navigation, and Control Conference, Toronto, Canada, August 2010.

[19] J. Russell Carpenter and K. T. Alfriend, "Navigation accuracy guidelines for orbital formation flying," Journal of the Astronautical Sciences, vol. 53, no. 2, pp. 207-219, 2005.

[20] U. Tancredi, A. Renga, and M. Grassi, "GPS-based relative navigation of LEO formations with varying baselines," in Proceedings of the AIAA Guidance Navigation and Control Conference, Toronto, Canada, August 2010.

[21] U. Tancredi, A. Renga, and M. Grassi, "Carrier-based Differential GPS for autonomous relative navigation in LEO," in Proceedings of the AIAA Guidance, Navigation, and Control Conference, Minneapolis, Minn, USA, August 2012, AIAA 20124707.

[22] U. Tancredi, A. Renga, and M. Grassi, "Ionospheric path delay models for spaceborne GPS receivers flying in formation with large baselines," Advances in Space Research, vol. 48, no. 3, pp. 507-520, 2011.

[23] A. Renga and M. Grassi, "Precise relative navigation for highly variable baselines using carrier-based differential GPS," in Proceedings of the 59th International Astronautical Congress (IAC '08), pp. 5000-5011, October 2008.

[24] A. Renga, M. Grassi, and A. Intelisano, "Carrier-based differential GPS filtering approaches for precise relative positioning in formation flying with highly variable inter-satellite distance," in Proceedings of the 3rd International Symposium on Formation Flying, Missions and Technologies (ESA-ESTEC '08), April 2008.

[25] P. de Jonge and C. Tiberius, The LAMBDA Method for Integer Ambiguity Estimation: Implementation Aspects, vol. 12, Delft Geodetic Computing Centre, 1996.

[26] A. G. Rodriguez, A.-M. B. Martinez, C. Mehlen et al., "GNSS in space: part 2 formation flying radio frequency techniques and technology," InsideGNSS, pp. 43-51, January-February 2009.

[27] G. Purcell, D. Kuang, S. Lichten, S.-C. Wu, and L. Young, "Autonomous formation flyer (AFF) sensor technology development," Technical Report NASA, TMO Progress Report 42$134,1998$.

[28] M. Aung, G. H. Purcell, J. Y. Tien et al., "Autonomous formation flying sensor for the starlight mission," in Proceedings of the International Symposium Formation Flying Missions and Technologies, Toulouse, France, October 2002.

[29] http://nssdc.gsfc.nasa.gov/multi/tdrs.html.

[30] S. Wang and E. Zhang, "Inter-satellite radio links and spreadspectrum ranging for satellite formation flying," in Proceedings of the 3rd International Conference on Microwave and Millimeter Wave Technology, pp. 233-236, August 2002.

[31] J. C. Adams, W. Gregorwich, L. Capots, and D. Liccardo, "Ultrawideband for navigation and communications," in Proceedings of the IEEE Aerospace Conference, vol. 2, pp. 785-792, Big Sky, Mont, USA, March 2001.

[32] F. D. Busse, Precise formation-state estimation in low earth orbit using carrier differential GPS [Ph.D. dissertation], Department of Aeronautics and Astronautics, Stanford University, Stanford, Calif, USA, 2003.

[33] J. A. Klobuchar, "Ionospheric effects on GPS," in Global Positioning System: Theory and Applications, B. W. Parkinson and J. J. Spilker, Eds., vol. 1, pp. 485-515, American Institute of Aeronautics and Astronautics, Washington, DC, USA, 1996.

[34] O. Montenbruck, M. Wermuth, and R. Kahle, "GPS based relative navigation for the TanDEM-X mission-first flight results," Navigation, vol. 58, no. 4, pp. 293-304, 2011-2012.

[35] http://www.gpsoftnav.com/satnav.html.

[36] A. Renga, U. Tancredi, and M. Grassi, "GPS-based relative navigation in earth observation missions relying on cooperative satellites," in Small Satellite Missions For Earth Observation, R. Sandau, H.-P. Roeser, and A. Valenzuela, Eds., pp. 467-475, 2010.

[37] ftp://cddis.gsfc.nasa.gov/gps/products/ionex/.

[38] O. Montenbruck and E. Gill, "Ionospheric correction for GPS tracking of LEO satellites," Journal of Navigation, vol. 55, no. 2, pp. 293-304, 2002. 

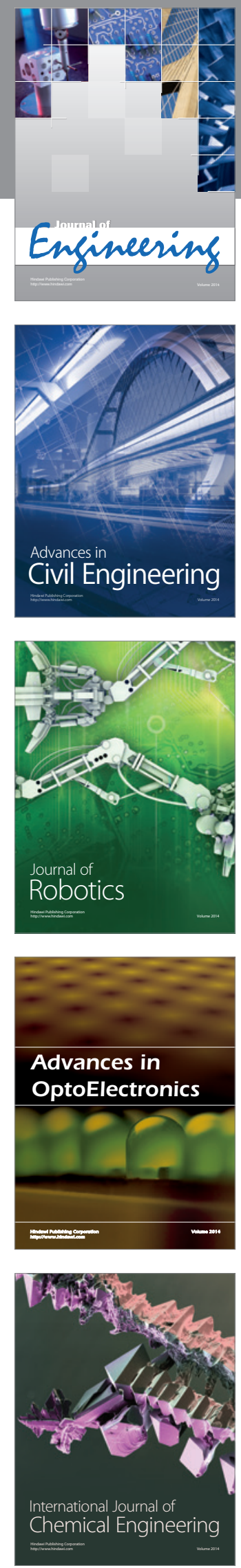

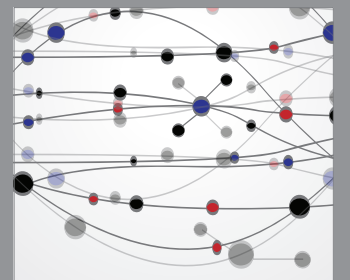

The Scientific World Journal
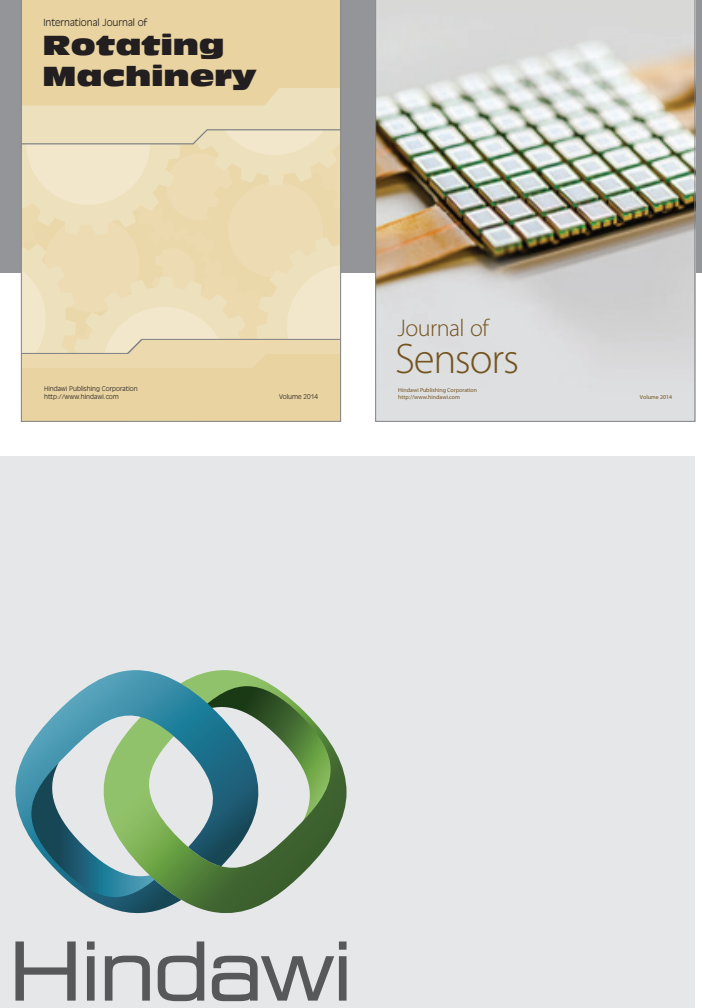

Submit your manuscripts at http://www.hindawi.com
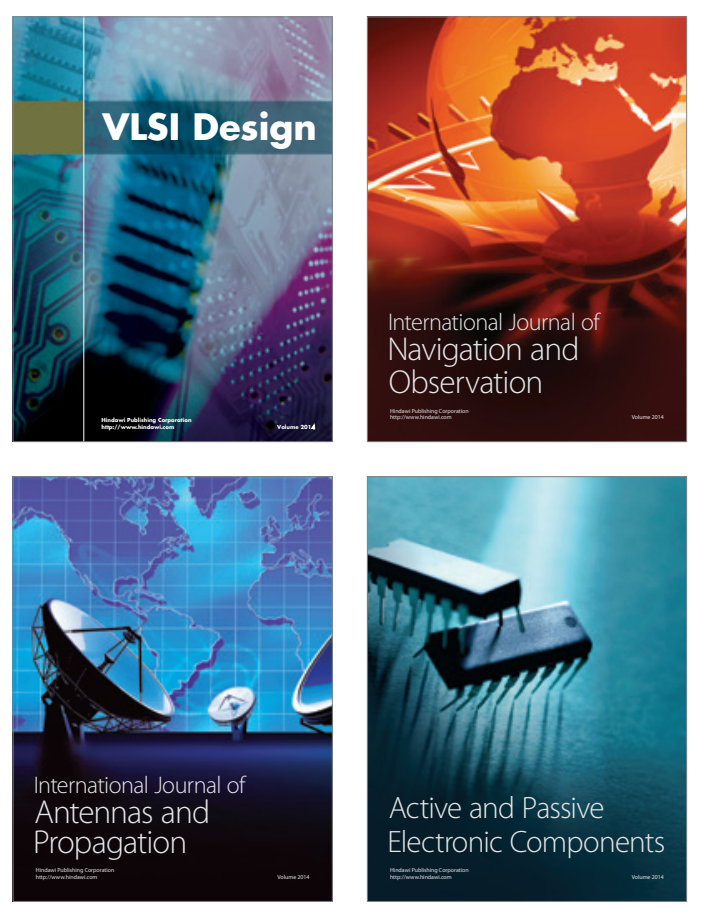
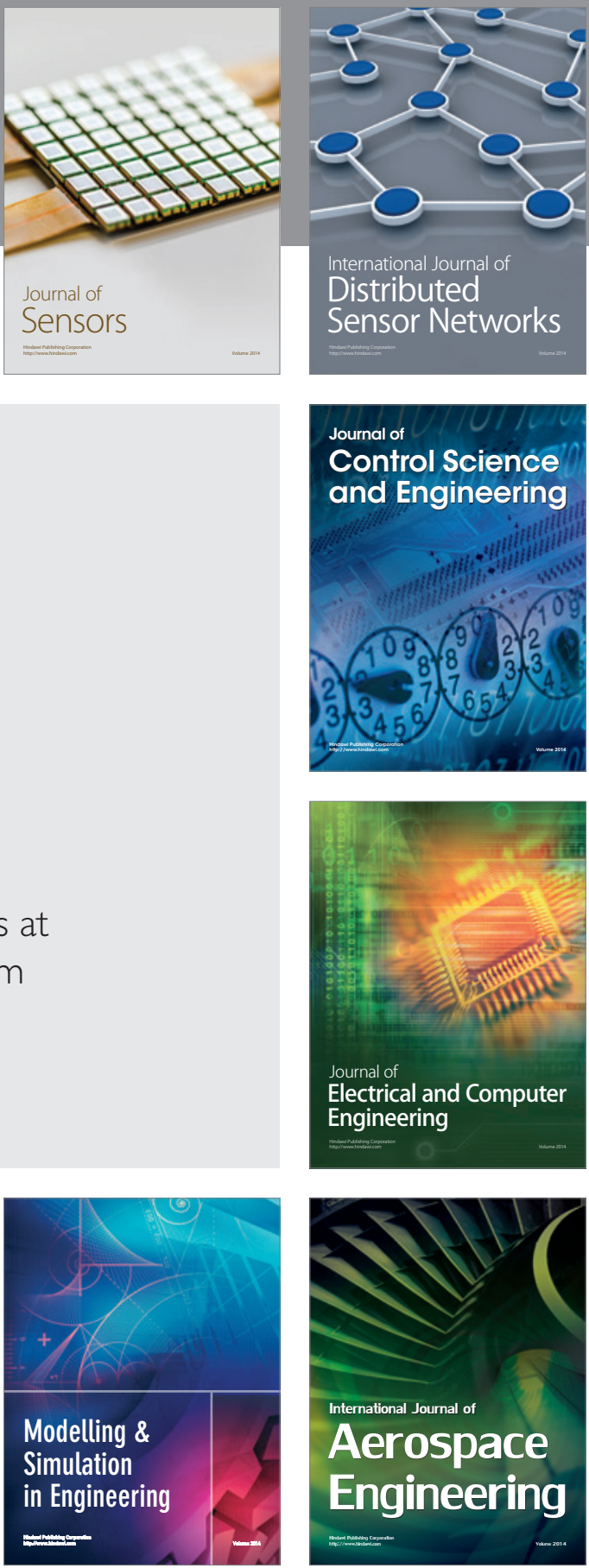

Journal of

Control Science

and Engineering
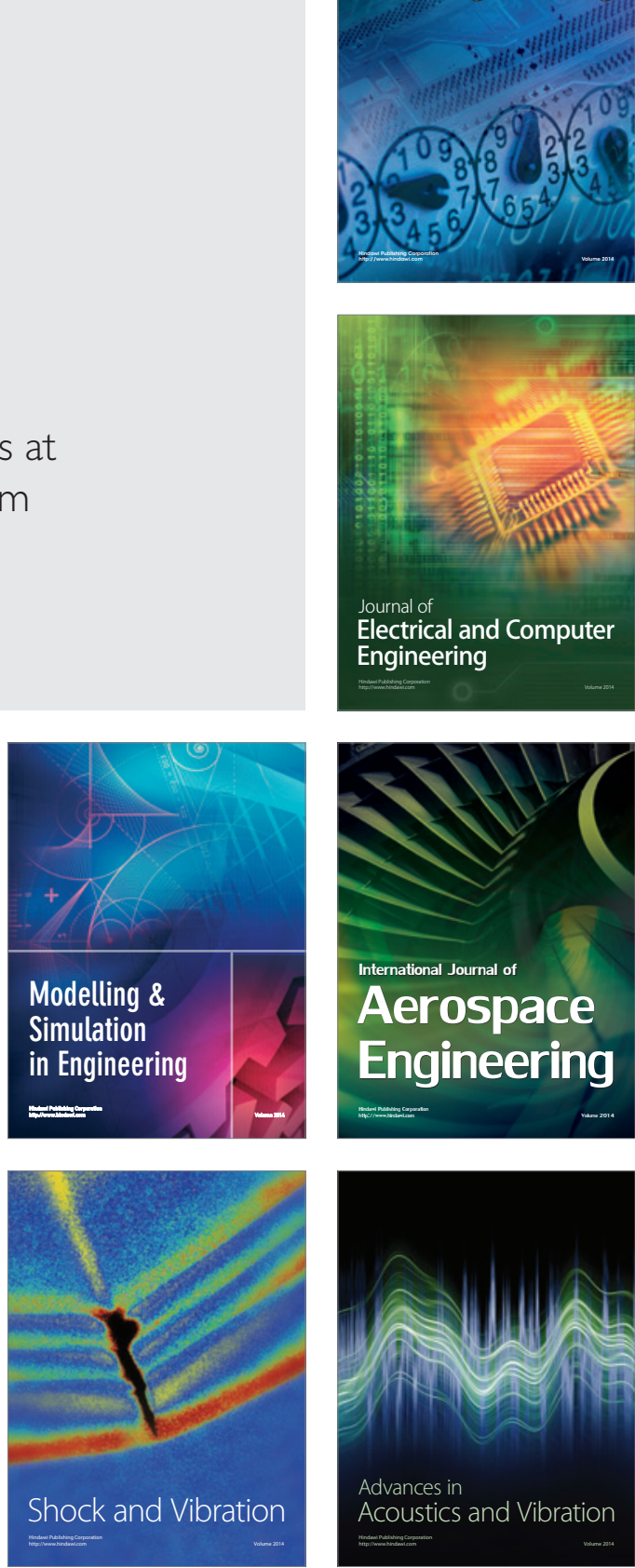